\title{
Automatic Artifact Removal from EEG - A Mixed Approach Based on Double Blind Source Separation and Support Vector Machine
}

\author{
Georg Bartels, Li-Chen Shi and Bao-Liang Lu* Senior Member, IEEE
}

\begin{abstract}
Electroencephalography (EEG) recordings are often obscured by physiological artifacts that can render huge amounts of data useless and thus constitute a key challenge in current brain-computer interface research. This paper presents a new algorithm that automatically and reliably removes artifacts from EEG based on blind source separation and support vector machine. Performance on a motor imagery task is compared for artifact-contaminated and preprocessed signals to verify the accuracy of the proposed approach. The results showed improved results over all datasets. Furthermore, the online applicability of the algorithm is investigated.
\end{abstract}

\section{INTRODUCTION}

Physiological artifacts in electroencephalography (EEG) recordings are a key problem in EEG-based brain-computer interfaces $(\mathrm{BCI})$ that aim at providing a non-muscular communication channel between human and machine. Such a system might help patients who are locked in their own bodies because of severe sicknesses to communicate with their surroundings. By analyzing certain features of their brain signals, one can enable them, e.g. to control the mouse cursor of a computer [1]. Unfortunately, the recorded EEG is often obscured by physiological artifacts - most prominently eye movement (electrooculography (EOG)) and muscle movement (electromyography (EMG)) artifacts. These unwanted signals make analysis of the recordings much more difficult and can even be mistaken for the physiological phenomena of interest - thus, eventually driving the BCI system [2].

Early studies used techniques of avoiding and rejecting artifactual signal trials to handle artifacts. These approaches are simple but might yield a huge loss of data. More advanced is the linear filtering method. This technique, however, is also prone to loss of information. Linear regression works well for EOG artifacts, but it is not applicable to remove EMG artifacts because it needs a reference channel. For a thorough and detailed review of the impact of artifacts on EEG-based BCI research and methods please refer to [3].

The most promising approach is to use blind source separation (BSS) techniques to separate the recordings into uncorrelated components [4] [5]. There are a lot of algorithms

This work was partially supported by the National Natural Science Foundation of China (Grant No. 60773090 and Grant No. 90820018), the National Basic Research Program of China (Grant No. 2009CB320901), the National High-Tech Research Program of China (Grant No. 2008 AA02Z310 and Grant No. 2008AA02Z315), and the Science and Technology Commission of Shanghai Municipality (Grant No. 09511502400)

G. Bartels, L. C. Shi and B. L. Lu are with the Center for Brain-Like Computing and Machine Intelligence, Department of Computer Science and Engineering, Shanghai Jiao Tong University and MOE-Microsoft Key Laboratory for Intelligent Computing and Intelligent Systems, Shanghai Jiao Tong University, 800 Dong Chuan Road, Shanghai 200240, China.

${ }^{*}$ Corresponding author (bllu@cs.sjtu.edu.cn). for BSS that all employ the same underlying model: The $M$ recorded EEG signals $\mathbf{x}(t)=\left[x_{1}(t), x_{2}(t), \cdots, x_{M}(t)\right]^{T}$ are assumed to be linear mixtures of the underlying $N$ components $\mathbf{s}(t)=\left[s_{1}(t), s_{2}(t), \cdots, s_{N}(t)\right]^{T}$ :

$$
\mathbf{x}=\mathbf{A s} .
$$

In general, the number of sources $N$ and the mixing matrix A are unknown. For simplicity of description, it is usually assumed that $M=N$. The recordings are separated into the components with the help of the unmixing matrix $\mathbf{W}$ (with $\mathbf{A} \approx \mathbf{W}^{-1}$ ) that has been calculated by the BSS algorithms:

$$
\mathbf{s}=\mathbf{W} \mathbf{x} .
$$

It is further assumed that in the course of this process artifacts and wanted EEG signals are separated into distinct components. Thus, one just has to identify the artifactual ones, and perform signal reconstruction without them, i.e. setting the corresponding rows of $\mathbf{W}$ to 0 , to obtain artifactfree data. This identification is usually done by manual inspection which is time-consuming and possibly biased [3], [6]. It has been shown that this approach can successfully separate EEG signals from EOG [7] and EMG [8] artifacts. Of the few presented automatic systems a lot either focussed on EOG only, or did not consider online applicability [9].

This paper presents an algorithm that has been designed to meet those needs: An universal preprocessing filter that automatically and reliably removes EOG and EMG artifacts from EEG recordings without the need for further visual inspection. The automatic design is based on two different source separation algorithms and support vector machine (SVM). Furthermore, we performed benchmarks to demonstrate that the proposed algorithm is also online-applicable.

\section{METHODS}

\section{A. Recording of Training Data}

In order to obtain recordings for SVM training, eight healthy male subjects between 21 and 29 were asked to perform 11 movements that generate various kinds of EOG and EMG artifacts of different magnitude: Head bending, teeth clenching, forehead movement, head turning, swallowing, eye blinking, eye rolling, making a fist, arm stretching, leg stretching, and finally performance of a random movement that the subject wanted to do at that moment.

The sessions were recorded using a 64-channel NeuroScan system. The recordings were done at a sampling frequency of $1 \mathrm{kHz}$, using an activated highpass filter at $0.01 \mathrm{~Hz}$, lowpass filtering at $100 \mathrm{~Hz}$, and a notch filter at $50 \mathrm{~Hz}$ to suppress 


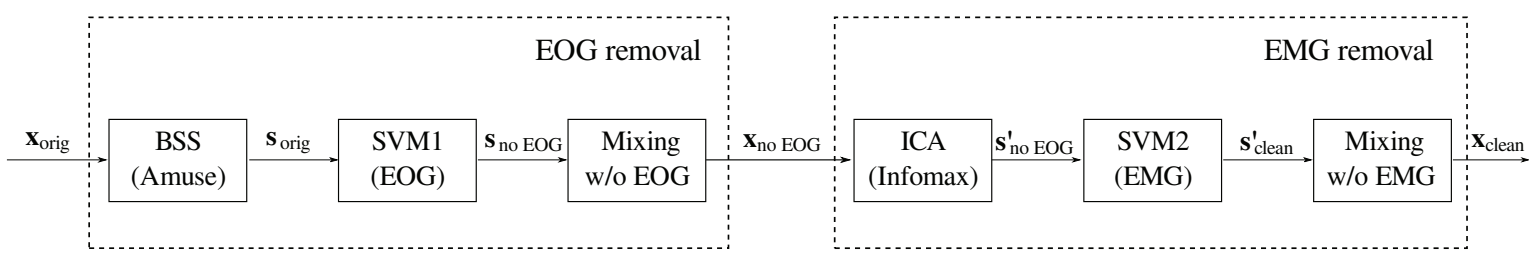

Fig. 1. Block diagram of the proposed artifact removal algorithm

power line noise. To shorten computation time the recorded data have later been downsampled to $250 \mathrm{~Hz}$.

The stimuli to perform the different movements were presented in random order - 20 times for each artifact. The structure of one trial was as follows: A short $1 \mathrm{sec}$ long phase of black screen was followed by a screen that said "attention" - also for $1 \mathrm{sec}$. Then the instruction to generate the specific artifact was presented for $7 \mathrm{sec}$. Finally, each trial ended with a short black screen for relaxation of random length between 2-4 sec. The recordings were done in 4 sessions, each lasting approximately $12 \mathrm{~min}$, with a $5 \mathrm{~min}$ in between. Thus, resulting in more than 100000 components for SVM classifier training.

In order to use the components that were extracted from the recordings for training the SVM classifiers, they had to be visually inspected and labeled into 3 classes: EOGcontaining, EMG-containing, or artifact-free ones. Recordings that were heavily contaminated with artifacts often yielded a lot of components that contained artifacts but also significant neural activity. In order to avoid loss of important EEG information only components with very strong EOG or EMG signals were labeled as such. As a result, one has to accept the risk of not removing all of the artifactual activity from the EEG - a trade-off between removing unwanted artifacts and preserving desired information that always has to be considered when performing artifact removal.

\section{B. Choice of BSS algorithm}

Choosing the right BSS algorithm for source decomposition in artifact removal applications and evaluating the quality of the yielded components is a rather difficult task. We applied two different algorithms: Infomax (an independent component analysis (ICA) algorithm) from the EEGLAB toolbox [10], and Amuse (BSS algorithm) from the ICALAB toolbox [11]. The main difference between these two algorithms is that Infomax yields truly statistically independent components, while Amuse just returns uncorrelated signals. For Infomax a step-limit of 32 was selected to limit computation to a reasonable amount. During labeling it became apparent that Amuse extracted EOG artifacts from the original recordings much better than Infomax: The components contained less neural activity and were also fewer in numbers for each decomposition. On the other hand, the performance of EMG-contaminated trials by Infomax was superior to that of Amuse. Thus, it was decided to incorporate both algorithms into the final design: Amuse to extract EOG artifacts, and Infomax to reliably obtain high-quality EMG components.

\section{Classification of Artifact Components}

Feature extraction was done for the 7 sec blocks of artifact performance. Three sets of features were used: component location, spectral information, and time-series information. Welch's algorithm was applied to calculate the power spectrum density (PSD) from 1 to $50 \mathrm{~Hz}$. Subsequently, the PSD was used to calculate the relative energy in $5 \mathrm{~Hz}-$ wide frequency bins - thus yielding 10 such bins. Spectral information is particularly useful for the classification task at hand, because EOG and EMG have typical spectra that often discriminate them from each other and the desired neural activity - with EOG showing much more energy at lower frequencies and EMG artifacts typically contaminating the entire frequency range of interest. The elements of the calculate mixing matrix $\mathbf{A}$ represent the topography of the components and were therefore also used as features. These are particularly useful for identifying EOG artifacts as these obviously often originate at frontal head regions. Finally, time-series information were incorporated by using autoregressive (AR) model parameters to detect sudden highamplitude changes in the signals [12]. With AR models one faces the problem of choosing the appropriate model order. A model of size 6 was selected, as this value minimized the error of the classification rate during 10-fold cross-validation (CV).

Classification of the EOG and EMG artifacts using the described feature vector was done with an SVM classifier with RBF kernel [13] - one SVM classifier for each kind of artifact. The training sets contained 2000 randomly selected samples, with one half being artifact-contaminated and the other half artifact-free. 10-fold CV yielded a good classification accuracy of over $90 \%$ for both tasks.

\section{Design of the Filter}

Figure 1 depicts the block diagram of the proposed algorithm. Firstly, the original recordings $\mathbf{x}_{\text {orig }}$ are decomposed into their corresponding components $\mathbf{s}_{\text {orig }}$ by using Amuse,

$$
\mathbf{s}_{\text {orig }}=\mathbf{W}_{\text {Amuse }} \mathbf{x}_{\text {orig }} \text {. }
$$

Then these components are classified with the first SVM classifier into EOG and artifact-free ones. With the help of the probability estimates $p_{i}$ of the SVM classifier, an entity matrix $\mathbf{E}_{E O G}$ is constructed, where $\mathbf{E}_{E O G(i, i)}=0$ if $p_{i}$ is greater than a threshold-value $t_{E O G}$. This threshold value has to be chosen such that the measure of quality of the final application is maximized, i.e. classification rate of a BCI system that uses the artifact-free EEG recordings. In 
addition, this threshold value is necessary as most decomposed components will contain both, artifacts and desired neural activity. Therefore $t_{E O G}$ is a means of changing the bias of the whole preprocessing artifact filter either towards removing a lot of artifacts or preserving vital information. Thus, the EOG-free recordings $\mathbf{x}_{n o} E O G$ can be expressed as follows:

$$
\mathbf{x}_{n o E O G}=\mathbf{A}_{\text {Amuse }} \mathbf{E}_{E O G} \mathbf{s}_{\text {orig }} .
$$

For EMG removal this process is repeated, the only real difference being that Infomax is applied for source separation. Another entity matrix $\mathbf{E}_{E M G}$ with $\mathbf{E}_{E M G(i, i)}=0$ for $p_{i}>t_{E M G}$ is calculated. Putting all this together the final artifact-free recordings $\mathbf{x}_{\text {clean }}$ have the following form:

$$
\mathbf{x}_{\text {clean }}=\mathbf{A}_{\text {Infomax }} \mathbf{E}_{E M G} \mathbf{W}_{\text {Infomax }} \mathbf{x}_{\text {no EOG }} .
$$

Note that the unmixing matrices $\mathbf{W}_{\text {Amuse }}$ and $\mathbf{W}_{\text {Infomax }}$ have to be recalculated every $7 \mathrm{sec}$, as the topography of the underlying components can only be expected to be stable for short periods of time. This is the main difference between the proposed approach and traditional off-line methods.

\section{RESULTS AND DISCUSSION}

A short 16-channel subset of the raw EEG recordings from posterior head regions is shown in Fig. 2. Strong artifacts caused by muscle activity are visible across all channels. It can clearly be seen that such strong EMG disturbance masks the less energetic neural information and is very likely to render the corresponding trials useless for automatic information extraction.

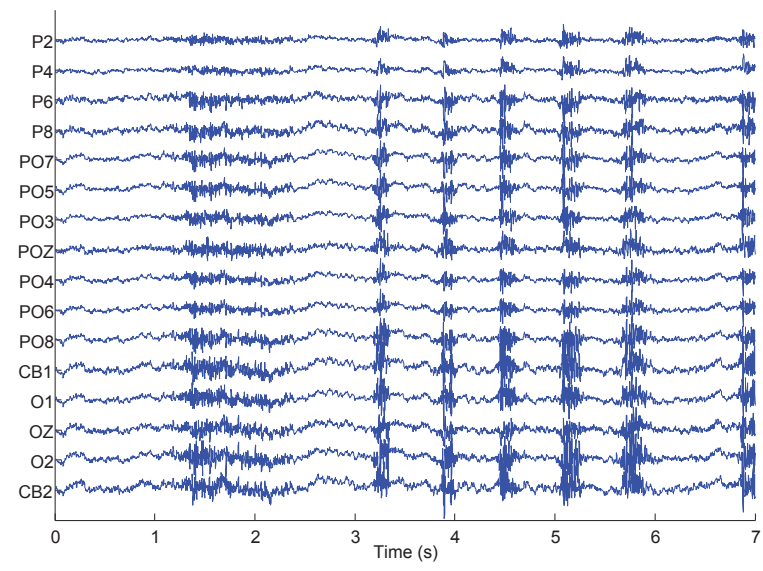

Fig. 2. Raw EEG recordings with strong muscle artifacts.

The signals from Fig. 2 after artifact removal are shown in Fig. 3. Obviously, most of the EMG artifacts that disturbed analysis of the original EEG recordings are now gone. Only small amounts of artifactual activity are still visible. Despite of the remaining small artifacts, the signal quality has improved dramatically. Moreover, when comparing the signals at the time steps without artifacts it becomes apparent that the desired original neural information remained almost

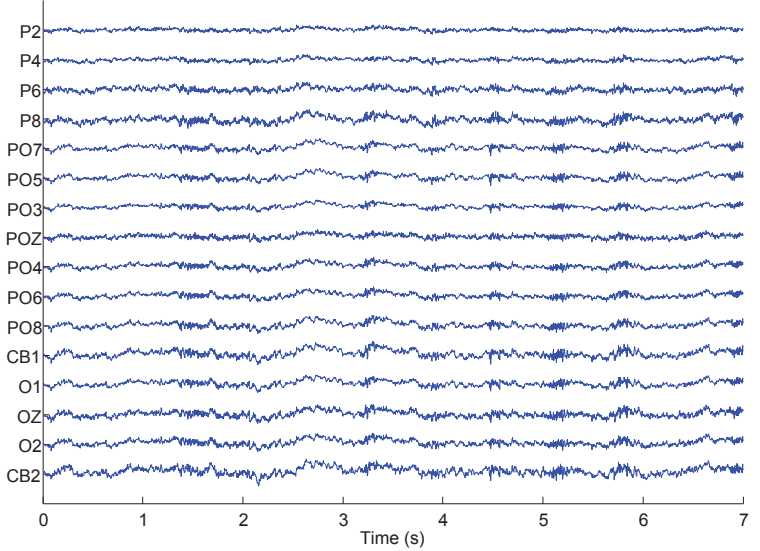

Fig. 3. Processed EEG after artifact removal (signals correspond to those depicted in Fig. 2).

untouched. Thus, the proposed approach can be a very effective and promising technique for artifact removal.

We further verified these results by comparing the classification rates on a two-class motor imagery task for preprocessed and unprocessed EEG signals. For this purpose the dataset IIIa from the BCI competition III (provided by Schlögl [14]) has been selected. Several reasons led to this choice. First of all, the recording settings between our experiment and Schlögl's experiment are very similar. The data were also recorded with a 64-channel Neuroscan amplifier using a sampling frequency of $250 \mathrm{~Hz}$ and it has been filtered between 1 and $50 \mathrm{~Hz}$. Unfortunately, only 60 channels were provided. Therefore the location feature could not be used for SVM classification, but 10-fold CV learning delivered only slightly downgraded performance (the classification rate is about $90 \%$ ) for this smaller feature vector. Secondly, the signals were also affected by various artifacts. Thus, the recordings are predestined for artifact removal.

The 4 classes of movements that had to be discriminated (left hand, right hand, tongue, and foot movement) were paired in 6 groups to yield the 2-class motor imagery datasets. To solve this task, common spatial pattern (CSP) and AR features were used for feature extraction and linear discrimination analysis (LDA) was employed for classification. The resulting classification performances are shown in Table I. The first column shows the results for the 2-class subsets of recorded data, while the last column holds the average classification rate over all subsets.

The performance for subject one $(\mathrm{k} 3 \mathrm{~b})$ did only improve slightly, which is not too surprising as the original average classification of $94.26 \%$ was already very high - apparently there were only little artifacts present in this recording. Considering this fact it is actually very good that the artifact removal did not downgrade the performance by unnecessarily removing wanted EEG activity from the signals. For the second subject $(\mathrm{k} 6 \mathrm{~b})$ the original classification was much worse with only an average success of $67.5 \%$. The data that have been processed by the proposed algorithm, on the other 
TABLE I

COMPARISON OF THE MOTOR IMAGERY PERFORMANCE FOR THE ORIGINAL DATA AND THE DATA AFTER ARTIFACT REMOVAL.

\begin{tabular}{l|c|c|c|c|c|c|c}
\hline Dataset & Task 1/2 & Task 1/3 & Task 1/4 & Task 2/3 & Task 2/4 & Task 3/4 & Average \\
\hline \hline k3b (orig) & $93.3 \%$ & $91.1 \%$ & $97.8 \%$ & $97.8 \%$ & $96.7 \%$ & $88.9 \%$ & $94.2 \%$ \\
k3b (clean) & $93.3 \%$ & $93.3 \%$ & $97.8 \%$ & $97.8 \%$ & $96.7 \%$ & $90.0 \%$ & $95.0 \%$ \\
\hline k6b (orig) & $58.3 \%$ & $55.0 \%$ & $68.3 \%$ & $65.0 \%$ & $66.7 \%$ & $91.7 \%$ & $67.5 \%$ \\
k6b (clean) & $61.7 \%$ & $63.3 \%$ & $81.7 \%$ & $66.7 \%$ & $66.7 \%$ & $93.3 \%$ & $72.2 \%$ \\
\hline 11b (orig) & $70.0 \%$ & $70.0 \%$ & $86.7 \%$ & $63.3 \%$ & $75.0 \%$ & $60.0 \%$ & $70.8 \%$ \\
11b (clean) & $75.0 \%$ & $75.0 \%$ & $86.7 \%$ & $70.0 \%$ & $88.3 \%$ & $66.7 \%$ & $77.0 \%$ \\
\hline
\end{tabular}

hand show improved results of almost 5 percentile. Thus, for a lot of trials the analysis of the original data was much more difficult due to present artifacts that masked the underlying neural activity of interest. However, the overall classification rate is still relatively low considering the simple task at hand. But this fact could also be caused by poor performance of the subject on the actual motor imagery task during recordings. The movement discrimination rate on the original dataset of the third and last subject (11b) was 3 percentile better than that for the preceding one: An average classification success of $70.8 \%$ was achieved. Again, the preprocessing to remove EOG and EMG artifacts yielded a great improvement in performance of more than 6 percentile. In summary, it is clear that the proposed algorithm is very effective when it comes to removing artifacts that have been caused by eye or muscle movement and still leaving valuable brain activity in the EEG mostly unchanged.

Furthermore, we investigated whether or not our artifact removal algorithm can be used for online applications. Therefore we ran several benchmarks on one of our servers (Intel Xeon E5440 with 8 Cores and $2.83 \mathrm{GHz}, 16 \mathrm{~GB}$ RAM, running Matlab 2009a using only a single core): Processing a $7 \mathrm{sec}$ long block of EEG recordings (64-channels, $250 \mathrm{~Hz}$ sampling frequency) took less than $4 \mathrm{sec}$. Therefore, it is possible to use the described approach for online usage if a delay of $4 \mathrm{sec}$ is acceptable for the application at hand.

\section{CONCLUSIONS}

In this paper, we have presented a new algorithm that uses BSS and SVM techniques to remove EOG and EMG artifacts from EEG recordings. In contrast to many other existing approaches, this is done automatically without the need for further human supervision. Additionally, the proposed algorithm is online applicable for applications that can accept a $4 \mathrm{sec}$ delay. Our experimental results on an independently recorded dataset for BCI motor imagery tasks verified that the preprocessing yields signals that contain much less artifacts and still hold the desired neural activity.

In the future, we will incorporate this algorithm into our real-time vigilance estimation system for driving simulation.
We expect to obtain improved overall application results for that task, too.

\section{REFERENCES}

[1] J. R. Wolpaw, N. Birbaumer, D. J. McFarland, G. Pfurtscheller, and T. M. Vaughan, "Brain-computer interfaces for communication and control," Clinical Neurophysiology, vol. 113, pp. 767-791, 2002.

[2] T. M. Vaughan, W. J. Heetderks, L. J. Trejo, W. Z. Rymer, M. Weinrich, M. M. Moore, A. Kübler, N. Birbaumer, E. Donchin, E. W. Wolpaw, and J. R. Wolpaw, "Brain-computer interface technology: a review of the Second International Meeting," IEEE Trans. Neural Syst. Rehab. Eng., vol. 11, pp. 94-109, 2003.

[3] M. Fatourechi, A. Bashashati, R. K. Ward, and G. E. Birch, "EMG and EOG artifacts in brain computer interface systems: A survey," Clinical Neurophysiology, vol. 118, pp. 480-494, 2007.

[4] T.-P. Jung, S. Makeig, C. Humphries, T.-W. Lee, M. J. McKeown, V. Iragui, and T. J. Sejnowski, "Removing electroencephalographic artifacts by blind source separation," Psychophysiology, vol. 37, pp. 163-178, 2000.

[5] R. Vigário, J. Särelä, V. Jousmäki, M. Hämäläinen, and E. Oja, "Independent Component Approach to the Analysis of EEG and MEG Recordings," IEEE Trans. Biomed. Eng., vol. 47, pp. 589-593, 2000.

[6] T.-P. Jung, S. Makeig, M. Westerfeld, J. Townsend, E. Courchesne, and T. J. Sejnowski, "Removal of eye activity artifacts from visual event-related potentials in normal and clinical subjects," Clinical Neurophysiology, vol. 111, pp. 1745-1758, 2000.

[7] C. A. Joyce, I. F. Gorodnitsky, and M. Kutas, "Automatic removal of eye movement and blink artifacts from EEG data using blind component separation," Psychophysiology, vol. 41, pp. 313-325, 2004

[8] M. Crespo-Garcia, M. Atienza, and J. L. Cantero, "Muscle Artifact Removal from Human Sleep EEG by Using Independent Component Analysis," Annals of Biomedical Engineering, vol. 36, pp. 467-475, 2008 .

[9] P. LeVan, E. Urrestarazu, and J. Gotman, "A system for automatic artifact removal in ictal scalp EEG based on independent component analysis and bayesian classification," Clinical Neurophysiology, vol. 117, pp. 912-927, 2006.

[10] A. Delorme and S. Makeig, "Eeglab: an open source toolbox for analysis of single-trial eeg dynamics including independent component analysis," Journal of Neuroscience Methods, vol. 134, pp. 9-21, 2004.

[11] A. Cichocki, S. Amari, K. Siwek, T. Tanaka, A. Phan, et al., "ICALAB toolboxes." [Online]. Available: http://www.bsp.brain.riken.jp/ICALAB

[12] T. Ning and J. D. Bronzino, "Autoregressive and bispectral analysis techniques: EEG applications," IEEE Engineering in Medicine and Biology Magazine, vol. 9, pp. 47-50, 1990.

[13] C.-C. Chang and C.-J. Lin, LIBSVM: a library for support vector machines, 2001, software available at http://www.csie.ntu.edu.tw/ cjlin/libsvm.

[14] A. Schlögl, "BIOSIG - an open source software library for biomedical signal processing." [Online]. Available: http://biosig.sf.net 